\title{
Las personas mayores y su salud: Situación actual
}

\section{Health status of the elderly. Current situation}

\author{
Ramos Cordero P*, Pinto Fontanillo JA**
}

\section{RESUMEN}

El envejecimiento de la población es un fenómeno que se está produciendo en todos los países desarrollados. España es el país de Europa con una mayor esperanza de vida y el segundo país a nivel mundial tras Japón. La previsión de este envejecimiento es que se mantenga una proyección constante y que afecte ponderalmente tanto al grupo de los mayores de 65 como al grupo de los mayores de 80, lo que se viene conociendo como el envejecimiento del envejecimiento o sobreenvejecimiento de la población. Las patologías crónicas como la hipertensión arterial, diabetes, dislipemias y obesidad presentan una tendencia ascendente. Se calcula que las enfermedades crónicas consumen el $80 \%$ de todas las consultas de Atención Primaria. Otro dato a tener en consideración es la ingesta de fármacos. El 88,9\% de los mayores de 65 años consume algún medicamento, llegando al 93,4\% cuando se consideran a los mayores de 75 años. El $94 \%$ de las personas que padecen enfermedades crónicas están polimedicadas.

Palabras clave: Envejecimiento, salud oral, salud general, personas mayores.

\section{SUMMARY}

All developed countries are facing ageing populations. Spain is the European country with the highest life expectancy; worldwide it is second only to Japan. This ageing is forecasted to continue, equally affecting people over 65 and those over 80, thus leading to what is known as the ageing of the ageing. Chronic pathologies such as hypertension, diabetes, dyslipidemia and obesity are steadily growing. Chronic diseases use up an estimated $80 \%$ of all Primay Care services. Medicine consumption should also be considered. $88,9 \%$ of those over 65 are taking some sort of drug; the percentage reaches $93,4 \%$ for those over 75 and $94 \%$ for people suffering from chronic diseases.

Key words: Ageing, oral health, systemic health, older adults.

Fecha de recepción: 2 de mayo 2015.

Aceptado para publicación: 6 de mayo 2015.

Ramos Cordero P, Pinto Fontanillo JA. Las personas mayores y su salud: Situación actual. Av. Odontoestomatol 2015; 31 (3): 107-116.

* Médico Especialista en Geriatría. Máster en Gestión y Administración Sanitaria. Coordinador Médico Asistencial del Servicio Regional de Bienestar Social. Consejería de Asuntos Sociales. Comunidad de Madrid. Secretario General de la Sociedad Española de Geriatría y Gerontología.

** Técnico Superior de Salud Pública. Responsable del Programa de Mayores. Subdirección General de Prevención y Promoción de la Salud. Dirección General de Atención Primaria. Servicio Madrileño de Salud. Comunidad de Madrid. 


\section{INTRODUCCIÓN}

El envejecimiento de la población es un fenómeno que hemos de contemplarlo como un éxito o logro de la salud en general y de la salud pública en particular. Si bien, es cierto que, a menudo, su grado de desarrollo y sus efectos se observan con cierta preocupación por los distintos responsables de su gestión, en la medida que plantean un reto para el sistema sanitario y para la sociedad en su conjunto (1).

En la actualidad, España es el país de Europa con una mayor esperanza de vida y el segundo país a nivel mundial tras Japón, un objetivo al que se ha llegado en gran medida a través de las estrategias sanitarias que se han venido incorporando en las últimas décadas según queda constancia en el Tercer Informe sobre Demografía publicado por la Comisión Europea de Estadísticas (Eurostat) (2).

Para comprender mejor esta situación es conveniente echar una mirada a su evolución histórica y en tal sentido encontramos que a principios del siglo XX, más de la mitad de la población se hallaba por debajo de los 24 años y su esperanza media de vida al nacer se situaba en torno a los 30-35 años de promedio. En la actualidad, un siglo después, más de la mitad de la población se sitúa en los 39 años, pero con una tendencia verdaderamente progresiva que nos advierte que en 2020 puede ser ya de 43 años y que, si sigue la tendencia, en 2049 la mitad de la población se situará en el límite de los 47 años. Consiguientemente la población se configura en una serie de pirámides progresivas que nos dejan esa imagen estructural de un envejecimiento cada vez más evidente y consolidado (Fig. 1).

Para desarrollar la presente división nos planteamos dividir el problema en varios apartados:

1. Evolución de la población mayor.

2. Esperanza de vida.

3. Tendencias.

4. Mortalidad: hacia un nuevo patrón.

5. Carga de enfermedad.

6. Uso del sistema sanitario.

7. Algunos indicadores positivos en los mayores de cara al futuro.



Fig. 1. Evolución de la población española. Pirámides de 2010, 2020 y 2049. Fuente: INE/INEBASE. Proyecciones de población. Base censo 2001

\section{EVOLUCIÓN DE LA POBLACIÓN MAYOR}

Especial relevancia tiene la proporción e influencia de las personas mayores en el conjunto global de la población. Según datos del Censo de Población y Viviendas de Noviembre de 2011 del INE (6), actualmente 8.116.347 personas tienen 65 años o más, lo que representa al $17,3 \%$ de la población, es decir, que tenemos una población muy envejecida. Dentro de este colectivo, casi la tercera, 2.438 .535 personas, es decir un 5,2\% del total de la población española, tienen 80 años o más, con una clara tendencia demográfica hacia la feminización de la población $(59 \%$ de mujeres y $41 \%$ de hombres). Más significativo aún es el dato de su evolución demográfica que se halla en torno al 3\% en cada una de las últimas décadas y que puede mantener ese crecimiento ya un poco atenuado del $2 \%$ hasta la mitad del presente siglo (1).

La previsión de este envejecimiento es que se mantenga una proyección constante y que afecte ponderalmente tanto al grupo de los mayores de 65 como al grupo de los mayores de 80 , lo que se viene conociendo como el envejecimiento del envejecimiento o sobreenvejecimiento de la población: un fenómeno de impacto todavía inevaluable (Fig. 2).

En concreto, lo esperable es que, a mediados del siglo, las personas mayores constituirán algo más del $32 \%$ de la población, o en términos absolutos, en torno a los 15 millones de personas. Se estima que en el año 2052, en España, habrá más de 15 millo- 


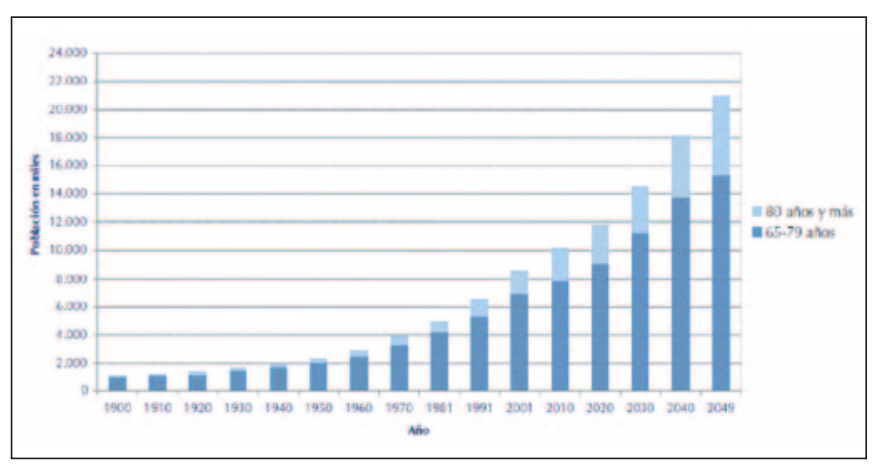

Fig. 2. Evolución de la población e personas mayores 1900-2049. Fuente: INE/INEBASE. Proyecciones de población a largo plazo. Febrero 2010.

nes de personas con 65 años o más, lo que representará el $37,2 \%$ de su población $(1,3)$.

Muchos son los factores que han permitido una evolución de esta naturaleza, singularmente la mejora en los hábitos de vida, el seguimiento y control de las enfermedades crónicas y la mejora de la atención sociosanitaria en general. De una forma más directa debe relacionarse con la caída de la mortalidad lo que converge en el aumento de la esperanza de vida como indicador global.

Paralelamente se producirá otro fenómeno de ajuste y es que España parece que empieza a estabilizarse demográficamente e incluso a perder población a partir del 2045. Las previsiones en forma de proyección del INE sugieren que habrá un descenso paulatino, habiendo llegado entonces a los 48 millones de habitantes. Este descenso desequilibra aun más la estructura de las edades y se espera que ni la natalidad ni el proceso inmigratorio neutralicen las cifras de mortalidad $(1,3)$.

\section{ESPERANZA DE VIDA}

Constituye un dato relacionado con una mejor salud relativa y, de manera más indirecta, con la propia calidad de vida (2).

En Europa, este indicador ha evolucionado positivamente especialmente en las últimas décadas y se reconoce a España como uno de los paises más destacados (2) (Eurostat 2011). La esperanza de vida para la mujer española ha alcanzado los 84,1 años, ocupando el segundo lugar, mientras que para el hombre es de 77,8, lo que le sitúa en el quinto lugar. Estos mismos datos, recogidos en nuestro país por el INE (referidos al Movimiento Natural de la Población 2011), ofrecen unas cifras aún mayores, con una esperanza de vida para las mujeres de 85,21 años y de 79,3 años para los hombres. Esto representaría la mayor expectativa de longevidad femenina de la UE y la tercera masculina (sólo detrás de Suecia e Italia) $(2,3)$.

Aunque sigue siendo notoria la diferencia por sexos, se constata una progresión constante sobre estos datos, sea cual sea la fuente, lo cierto es que las proyecciones vienen también a poner de manifiesto una cierta suavización o ralentización de esas ganancias. Del mismo modo y de forma paralela se observa un acortamiento progresivo de la marca diferencial entre hombres y mujeres, lo que se achaca a una serie de factores comportamentales que convergen en una evidenciable superior reducción de la mortalidad masculina en comparación con la femenina, si bien siga siendo aun más alta. Al día de hoy, el trecho de la esperanza de vida por sexos es de 6,1 años y se espera que vaya suavizándose hasta los 5,6 años en $2049(1,2)$.

Más sensible es el indicador que mide la esperanza de vida una vez llegados a la teórica marca de los 65 años. Actualmente la expectativa para las personas de 65 años es que puedan vivir una media de 20,5 años más. A las mujeres se le atribuye una esperanza adicional de 22,3 años y al hombre de 18,4 según el INE (Movimiento Natural de la Población, 2011) (1). Este dato incide aún más en el encabezamiento de la esperanza de vida de la población mayor española en Europa y también en el resto del mundo.

Y, más sensible aún, si lo relacionamos con el verdadero estado de salud y de la llamada calidad de vida, es el índice de esperanza vida libre de incapacidad al cumplir los 65 años que nos dice que para las mujeres es de 10 años y algo más, 10,3 para los hombres. Por lo tanto, la situación resultante es que las mujeres tienen un horizonte de vida en condiciones de incapacidad de 11 años y los hombres de 7 (1,35). Es decir, la opción de una vida larga no se corresponde en nuestro país con la realidad de una vida en 
buena salud (3-5). Quiere esto decir lo que ya se ha puesto de manifiesto por diferentes sociedades científicas, en particular con las relacionadas con la atención a los mayores como la Sociedad Española de Geriatría y Gerontología, cuando alertan de que en España debe hacerse hincapié en promover un envejecimiento de más calidad. A este fenómeno es especialmente sensible la mujer mayor que vive esta etapa con más carga de enfermedad, dolor y discapacidad las mujeres $(1,6)$.

\section{TENDENCIAS}

Dando por hecho que el envejecimiento poblacional es la tendencia guía a la que hay que prestar una atención genérica, no es menos relevante la cuestión de los desequilibrios demográficos que se constituyen en subtendencias y que tienen una importancia considerable en futuro de inequidad global. La llamada "reestructuración de las edades" subsiguiente al proceso de envejecimiento altera profundamente las relaciones intergeneracionales en lo que se refiere a la convivencia, la solidaridad y los cuidados y atenciones mutuas. Entre estas evidencias, cabría destacar, al menos, estos dos:

a) La primera subtendencia observable en España, así como en los países del entorno y que tiene cierto carácter de emergente es la inversión demográfica. Es el fenómeno que se da en la actualidad y por primera vez y que se cifra en que la población de personas de 65 años y más supera en número a la de los niños de 0 a 14 años (Fig. 3). El crecimiento del primer grupo y el decreci-

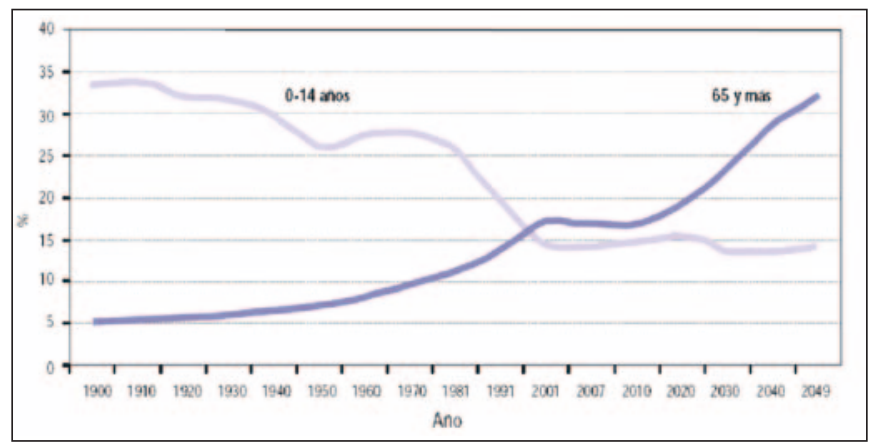

Fig. 3. Inversión de la tendencia demográfica. Fuente: INE/INEBASE. Datos reales, 1900 a 2007. INE/INEBASE. Proyecciones de población a largo plazo, 2009-2049. miento del segundo confluyen en un punto crítico que cuanto más se acentúa más pone en peligro la sostenibilidad del sistema de protección sociosanitaria (1,3-6).

b) La segunda subtendencia recoge el hecho de la reducción diferencial progresiva de esperanza de vida entre hombres y mujeres mayores a partir de los 65 años. Si el índice general disminuía de 6,1 años a 5,6, tomando como referencia la edad de jubilación se invierte este patrón: pasa de 3,9 años actuales a 4,3 en 2049. Es decir vuelve a evidenciar un desequilibrio entre sexos entre las personas mayores que tendrá consecuencias sanitarias, sociales y vitales (1).

\section{MORTALIDAD: HACIA UN NUEVO PATRÓN}

Son apreciable cambios evidentes en la conformación del patrón de mortalidad actual. El más singular es que es asignable en una gran medida a personas mayores, habiéndose producido en este sentido un cambio de paradigma. Mientras que a principios del siglo pasado se adscribía preferentemente a niños de corta edad, a principios de este se adscriben a personas de edad avanzada. En un siglo, ha pasado del $30 \%$ al $85 \%$ la proporción de fallecidos mayores en el conjunto de la población (3-5).

Por otro lado, se ha producido una cierta estandarización de fallecimientos en edades y procesos desencadenantes; en general se concentra en las edades muy avanzadas. Esto no es óbice para que, en general, se constate un descenso de la mortalidad en todas las edades, también en lo que atañe a las personas mayores, lo que supone longevidad añadida o sobreenvejecimiento; una situación sostenible previsiblemente en el tiempo. Así, la tasa de mortalidad de la población española era de 8,4 por mil en el año 2008, mientras que sólo la de 65 y más era de 42,1 por mil, pero habiendo descendido con respecto a una década anterior en 8 puntos (IMSERSO, Libro Blanco, 2011) y a comienzos de siglo era del 21,9 por mil $(4,5)$.

La mortalidad en relación al sexo se mantiene en la vejez con predominio masculino (algo que está desapareciendo en el resto de edades), consolidando el diferencial y la resultante de más mujeres mayores 
solas a cierta edad, tanto en la casa como en las instituciones. En cuanto a las causas de muerte, se ve un cambio de tendencia muy notable desde las últimas décadas (Fig. 4).

En este cambio se observa como hecho más significativo el que las enfermedades degenerativas toman el relevo y desbancan a otras más tradicionales, de relevancia histórica, preferentemente agudas y contagiosas (1). En este cambio se ven inmersos también los mayores (Fig. 5).

El patrón de mortalidad predominante en las personas mayores por grandes grupos de enfermedades viene definido por las enfermedades del sistema circulatorio (30,5\% del total de defunciones), los tumores $(28,2 \%)$ y las enfermedades del sistema respiratorio (10,9\%). Manteniendo la tendencia de los últimos años, ascendente para los tumores (2,0\% más de fallecidos) y descendente para las enfermedades circulatorias $(0,7 \%$ menos de fallecidos) e irrumpiendo as-

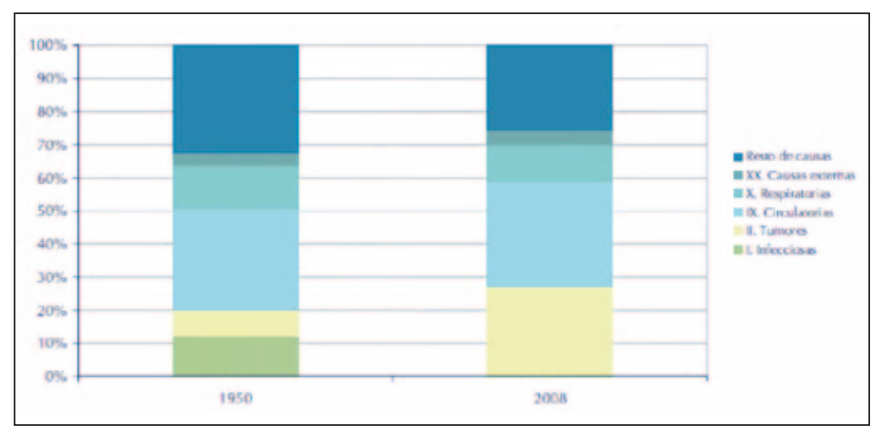

Fig. 4. Defunciones y causas, 1950 y 2008. Fuente: INE/INEBASE. Defunciones por causa de muerte. Tablas nacionales. 2011.

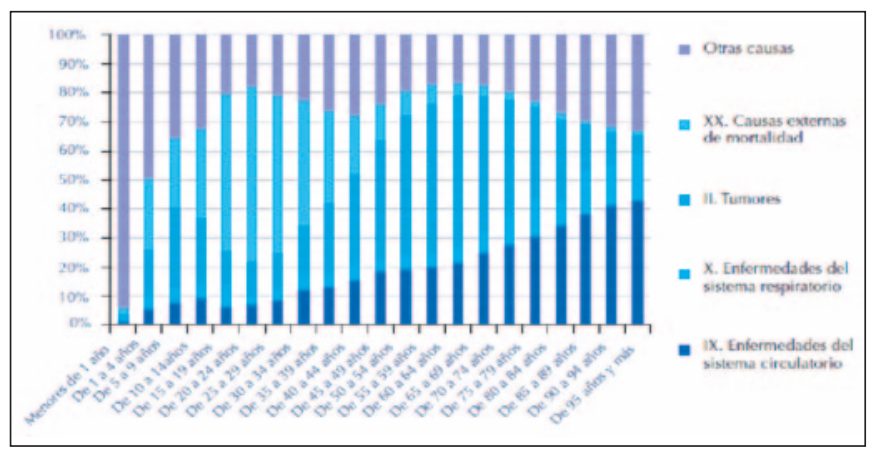

Fig. 5. Defunción por causa y grupo de edad. 2008-11. Fuente: INE/ INEBASE. Defunción por causa de muerte 2011. cendentemente las muertes por enfermedades respiratorias $(4,9 \%$ más de fallecimientos) $(1,3)$.

Dentro de las principales causas de muerte, éstas varían según el sexo; siendo los tumores la primera causa de muerte en los hombres $(2,95 \%$ ) y la segunda en mujeres (1,8\%o. Dentro de éstos, el cáncer de bronquios y pulmón es el que más muertes produce en hombres frente al cáncer de mama en las mujeres. Por el contrario, en las mujeres, la primera causa de muertes son las enfermedades circulatorias, las cuales han descendido respecto al año anterior.

En las últimas décadas, se observa un incremento verdaderamente significativo atribuible a las enfermedades del sistema nervioso $(5,0 \%)$, hasta convertirse en la cuarta causa de muerte más frecuente, especialmente las enfermedades neurodegenerativas cuyo principal protagonista viene definido por la demencia tipo Alzheimer con 11.907 muertes, (más del doble de fallecimientos que en el año 2000, posiblemente fruto de una mejor codificación de las causas de muerte) $(1,3)$. La más afectada es la mujer en tanto que es más superviviente en las edades más avanzadas (Fig. 6).

Los fallecidos por accidentes de tráfico se han reducido respecto al año 2010 a 2.116 personas (un 9,4\% menos). Desde comienzos de la década anterior las muertes por accidente de tráfico se han reducido aproximadamente a la tercera parte.

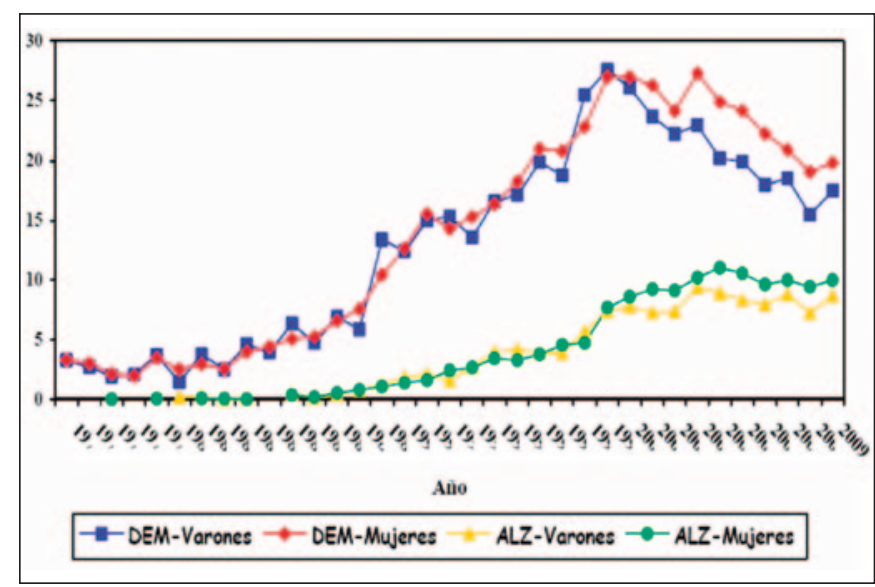

Fig. 6. Evolución de la mortalidad por demencia y enfermedad de Alzheimer según sexo. Comunidad de Madrid. 1975-2007 por 100.000 habitantes/año estandarizada por edad y sexo. 
Sabemos que las enfermedades crónicas constituyen la principal causa de mortalidad en el mundo, representando hasta el 63\% de las muertes anuales y se estima que en los próximos años esta proporción se elevará alcanzando casi al $80 \%$. Así, en el año 2008 según la OMS, 36 millones de personas murieron como consecuencia de una enfermedad crónica y, de éstas, unos 27 millones se concentraron en los mayores de 60 años. En España, el 84,9\% de las muertes acontecen en personas de 65 años o más $(7,8)$.

Según los últimos datos disponibles sobre mortalidad general en España, se han producido 387.911 defunciones, que arrojan una tasa bruta de mortalidad del 8,41\%o, 188.057 mujeres y 199.854 hombres. Por sexo, la tasa femenina se situó en $8 \%$ on mujeres y $8,8 \%$ o en hombres $(7,8)$.

\section{CARGA DE ENFERMEDAD}

El cambio anteriormente referido en el perfil o patrón de mortalidad en los mayores, viene determinado por el propio cambio en los patrones de morbilidad (enfermedad). Las enfermedades crónicas de tipo clásico junto con las enfermedades de carácter degenerativo se van imponiendo, en detrimento de las enfermedades infecciosas tradicionales y mucho más de las de tipo parasitario en franca retirada.

El patrón de morbilidad viene definido por las enfermedades de la "civilización" o del "progreso", fruto de determinados hábitos y estilos de vida como el sedentarismo, el estrés, la alimentación inadecuada y el consumo de tóxicos: alcohol, tabaco, drogas y medicamentos. Este patrón viene representado fundamentalmente por las enfermedades circulatorias $(21,4 \%)$, respiratorias $(15,5 \%)$ y el cáncer $(12,4 \%)$, seguidas de otras como la diabetes, las enfermedades neurodegenerativas, enfermedades digestivas, etc. $(7,8)$.

Se trata de enfermedades de carácter degenerativo, con tendencia a la cronicidad y a la asociación de enfermedades en una misma persona (pluripatología o comorbilidad), que le imprimen un cierto grado de fragilidad clínica, con deterioro funcional, pérdida de su autonomía y discapacidad y tendencia a la polimedicación (9). La carga de enfermedad hoy bascula entre dos tendencias: la relevancia de las enfermedades degenerativas y la mejora en el abordaje de la discapacidad. De esta forma, la pluripatología, la comorbilidad y la limitación de las funciones básicas de la vida diaria, han pasado a constituir la prioridad en el abordaje terapéutico más que la propia enfermedad aislada en sí misma. En este modelo o patrón de morbilidad, "la enfermedad aislada" deja de acaparar todo el protagonismo, para adquirir mayor relieve la comorbilidad y la limitación funcional que estas enfermedades imprimen al paciente (7-9).

La metodología de los cuidados, la rehabilitación, la propia fortaleza del sistema sanitario son, junto a la innovación en los tratamientos farmacológicos, los principales retos para alcanzar o preservar una mayor autonomía del paciente y un retraso de los fallecimientos.

La cronicidad está muy presente en las cortes de edad avanzada. Según datos de la Encuesta Nacional de Salud del año 2011-2012 (3), existe una tendencia ascendente de las patologías crónicas como la hipertensión arterial, diabetes, dislipemias y obesidad, todos ellos factores de riesgo cardiovascular, hasta el punto que el $73 \%$ de los mayores de 65 años declaran presentar al menos una enfermedad crónica, elevándose al 78,5\% en los mayores de 75 años $(77,3 \%$ de los hombres y $79,2 \%$ de las mujeres). Se estima que realmente, hasta un $95 \%$ de mayores, padece alguna dolencia de carácter crónico pese a que en algunos casos estos no las perciban como ocurre con la diabetes, dislipemia, cataratas, etc. (3-5).

La obesidad tiene una curva bimodal, siendo más frecuente a medida que avanza la edad, hasta los 74 años, momento en el que comienza a descender. Entre el 70 al $80 \%$ de las personas mayores padecen problemas cardiovasculares. La enfermedad pulmonar obstructiva crónica está presente entre el 20 y el $30 \%$ de los mayores. En torno al $90 \%$ presentan déficits sensoriales de algún tipo (4).

Un 53\% de las personas mayores tiene problemas articulares y periarticulares de tipo artrosis, artritis o problemas reumáticos. Esta proporción aumenta en las mujeres que además presentan más lesiones y problemas en general que cursan con dolor (3). 
La presencia de dolor, globalmente considerado y de cualquier etiología, es muy frecuente en las personas mayores, aumenta con la edad y en la mujer, encontrando que en el hombre lo padecen el $32 \%$ de las personas entre los de 65 a 74 años, asciende al $43 \%$ entre los de 75 a 84 años y hasta el $60 \%$ en los mayores de 85 años; mientras que en la mujer alcanza al 53\% entre las de 65 a 74 años, asciende al $65 \%$ entre las de 75 a 84 años y hasta el $75 \%$ en las mayores de 85 años (3).

En cuanto las enfermedades infecciosas y las tumorales, ven agravados sus pronósticos y, en consecuencia, su prevalencia al ser coadyuvados por otros procesos convergentes con el propio envejecer.

Por último, los accidentes también aumenta con la edad y en la mujer, encontrando que entre los 65 a 74 años sufren accidentes el 3,6\% de los hombres y el $10 \%$ de las mujeres, entre los 75 y 84 años aumentan al $4,5 \%$ en los hombres y al $12,1 \%$ en las mujeres y, en los mayores de 85 años, alcanzan al $10,2 \%$ de los hombres y al $14 \%$ de las mujeres (3).

Aún con este perfil de enfermedad descrito, sólo el $15 \%$ del conjunto de la población mayor considera su estado de salud como malo, o muy malo (5). Según datos de la Encuesta Nacional de Salud del año 2011-2012 (6) solo el 24,7\% de la población total cree padecer problemas en su estado de salud ( $20,7 \%$ en hombres y $28,7 \%$ en mujeres) y esta percepción va aumentando a medida que avanza la edad, encontrando que entre los 65 a 74 años alcanza $39,5 \%$ de los hombres y al $51,7 \%$ de las mujeres; entre los 75 a 84 años al $58 \%$ de los hombres y al $68,7 \%$ de las mujeres y para los mayores de 85 años llega hasta el $67,9 \%$ de los hombres y el $71 \%$ de las mujeres (3).

Del mismo modo la percepción de presentar algún problema de salud o enfermedad crónica, va aumentando con la edad y siempre con mayor carga de enfermedad en la mujer, encontrando que entre los 65 a 74 años lo perciben el 65,3\% de los hombres y el $70,8 \%$ de las mujeres; entre los 75 a 84 años asciende al $77,6 \%$ en los hombres y al $78 \%$ en las mujeres y en los mayores de 85 años alcanza al 76,8\% en los hombres y al $82,7 \%$ en las mujeres.
Respecto a los hábitos y estilos de vida solo un $59,1 \%$ de la población española reconoce que realiza alguna actividad física en su tiempo libre como caminar, practicar deporte, gimnasia etc. $(64,7 \%$ en los hombres y $53,8 \%$ en las mujeres).

En relación a los hábitos tóxicos, se observa que el consumo de tabaco va descendiendo con la edad y siempre es menor en la mujer, encontrando que entre los 65 y 74 años el 16,2\% de los hombres y el $4,6 \%$ de las mujeres son fumadores habituales, mientras que entre los 75 y 84 años baja al $8,9 \%$ en los hombres y $0,9 \%$ en las mujeres y en los mayores de 85 años desciende hasta el $4,4 \%$ en los hombres y el $0,5 \%$ en las mujeres (3).

Del mismo modo el consumo de alcohol, va descendiendo con la edad y el sexo, aunque aún ha de mejorar, ya que en el último año han consumido alcohol el $67,4 \%$ de los hombres y el $34,2 \%$ de las mujeres; presentando un consumo intensivo de al menos una vez al mes, el 3,2\% de los hombres y el $0,7 \%$ de las mujeres entre los 65 a 74 años y el $1,4 \%$ de los hombres y el $0,2 \%$ de las mujeres en los mayores de 75 años (3-5).

Estas enfermedades crónicas asociadas, tienden a producir diferentes grados de discapacidad afectando a la autonomía funcional. Así, encontramos que la autonomía funcional de la población fue descendiendo desde 1993 a 2006, tanto en hombres como en mujeres, y presenta ahora una ligera mejora. En 1993, el 62,9\% de la población de 65 y más años (70,1\% de los hombres y 58,0\% de las mujeres) era capaz de realizar sin ayuda las actividades de la vida diaria. En 2012, este porcentaje es del 53,5\% (61,3\% de los hombres y $47,7 \%$ de las mujeres), algo mayor que en $2006(51,4 \%)(8,9)$.

En lo que respecta a la permanencia en situación de autonomía, existen estudios que ponen en evidencia que se está produciendo un cierto retraso en la entrada en discapacidad y dependencia $(4,5)$ si bien aun no puedan considerarse del todo concluyentes por la metodología y muestras utilizadas.

A la luz de los datos de los que disponemos a fecha de 2012 , el $46,5 \%$ presenta algún problema de de- 
pendencia funcional y el 35\% de estos son dependientes para alguna actividad básica de la vida diaria (tales como vestirse, asearse, etc.), presentando un Índice de Barthel inferior a 60 puntos, siendo especialmente frecuente en los mayores de 80 años, en los que más del $50 \%$ presenta alguna discapacidad que le causa problemas para el desarrollo de las ABVD. En todos estos casos, globalmente considerados, la mujer presenta un mayor grado de dependencia que el hombre $(32,4 \%$ de las mujeres mayores reconocen limitaciones frente al $23,7 \%$ de los hombres), pero si se desdoblan por tareas, el hombre presenta una mayor dependencia para las tareas domésticas, mientras que la mujer para la movilidad (5).

Se estima que en un futuro la población dependiente alcanzará en 2020 en torno a 2.300 .000 personas en todos los grados (5) (Fig. 7).

\section{USO DEL SISTEMA SANITARIO}

En las últimas décadas, se ha triplicado la asistencia y utilización del sistema sanitario por paciente, pero con especial significación por parte del paciente mayor.

\section{a) Atención Primaria}

Según la Encuesta de Salud 2011-2012 (3) en el último año, han asistido a alguna consulta médica, el $88,1 \%$ de los hombres y el $92,3 \%$ de las mujeres



Fig. 7. Evolución del ratio de dependencia de la población española. $\%$ Personas $\geq 65$ años/\% personas activas (20 a 64 años). Fuente: INE/INEBASE. Censos y proyecciones de población a largo plazo 2010-2049. entre los 65 y 74 años, sube al 94,9\% de los hombres y al 95,9\% de las mujeres entre los 75 y 84 años y alcanza al $92,2 \%$ de los hombres y al 93,8\% de las mujeres en los mayores de 85 años, siempre con mayor frecuencia en la mujer. Del mismo modo, cuando se analiza la asistencia a la consulta médica en último mes, este fenómeno se reproduce, alcanzando en el grupo comprendido entre los 65 y 74 años un $39,25 \%$ en hombres y un $44,4 \%$ en mujeres; en las personas entre 75 y 84 años asciende al $49,8 \%$ en hombres y al 50,6\% en mujeres y, en los mayores de 85 años, desciende al 46,6\% en hombres y al $48 \%$ en mujeres (3).

En el año 2013, un 91,7\% del global de la población madrileña mayor de 64 años con derecho a asistencia sanitaria pública fue atendida en las consultas de Atención Primaria (muy similar a otras comunidades autónomas). La patología del aparato circulatorio, básicamente crónica, constituye el motivo principal de consulta en Atención Primaria en hombres (hipertensión arterial no complicada especialmente), mientras que las patologías del aparato locomotor, sobre todo agudas, son la primera consulta en mujeres (3).

Se calcula que las enfermedades crónicas consumen el $80 \%$ de todas las consultas de Atención Primaria $(7,8)$.

El 88,9\% de los mayores de 65 años consume algún medicamento, llegando al 93,4\% cuando se consideran a los mayores de 75 años y el $94 \%$ de las personas que padecen enfermedades crónicas están polimedicadas (9). Esto conlleva que las personas mayores que representan al 17,3\% de la población, consuman el $30-40 \%$ de la prescripción y el $70 \%$ del gasto farmacéutico del Sistema Nacional de Salud $(9,10)$.

Además sabemos que están polimedicados (consumo de forma continuada durante los últimos 6 meses, de 6 o más principios activos), siendo en no pocas ocasiones responsables en los mayores de la falta de cumplimentación y adherencia terapéutica, de reacciones adversas, de entre un $7-17 \%$ de los ingresos hospitalarios, e incluso algunos de esos eventos en un $2-3 \%$ de los casos se relacionan con la causa de la muerte (9). 


\section{b) Atención especializada}

En el nivel de Atención Especializada se observa un incremento de la frecuentación por causas y asignable en buena medida a las personas mayores. Sabemos que en España el $41,90 \%$ de todas las altas hospitalarias, se producen en personas de 65 años o más, con unas estancias medias hospitalarias globalmente consideradas, más prolongadas que en los adultos (9).

Las tasas de altas hospitalarias en personas de 65 años o más, han aumentado un $32,3 \%$ en el periodo 2003-2013. Parte de ese aumento puede deberse al aumento de longevidad en ese periodo. La patología del sistema nervioso y de los órganos de los sentidos y la del aparato circulatorio, son por este orden las dos primeras causas de alta hospitalaria en ambos sexos.

Actualmente, más del $50 \%$ de las estancias hospitalarias corresponden a personas de 65 y más años $(8,9)$.

Según la Encuesta Nacional de Salud del año 2011 2012 (3) los ingresos hospitalarios producidos durante el último año, en personas mayores de 65 años, aumentan con la edad y con el sexo (hombre); así encontramos que entre los 65 y 74 años han ingresado el $11,7 \%$ de los hombres y el 9,4 de mujeres; entre los 75 y 84 años han ingresado el 19,9\% de los hombres y el $14,8 \%$ de las mujeres y finalmente en los mayores de 85 años han ingresado el 22,5\% de los hombres y el 16,1\% de las mujeres (Fig. 8).



Fig. 8. Diagnósticos al alta en $\geq 65$ años/100.000 habitantes (ambos sexos). (1985-2009). Fuente. INE/INEBASE. Padrón municipal. 2009.

\section{ALGUNOS INDICADORES POSITIVOS EN LOS MAYORES DE CARA AL FUTURO}

- El 53\% de personas mayores inician nuevas actividades después de los 65 años.

- El $43 \%$ de los mayores manifiesta querer vivir activamente y de forma autónoma en la sociedad española (4).

- Las personas mayores expresan una clarísima voluntad de autonomía, como manifiesta el hecho de que un $87 \%$ desea vivir en su casa el mayor tiempo posible. No obstante, también desean fuertemente mantener relaciones familiares intensas, de las que ya disfruta el $89 \%$. Incluso un $70 \%$ contribuyen al cuidado de sus nietas y nietos.

- Un 28\% pertenecen a alguna organización y un $8 \%$, concretamente, a organizaciones de voluntariado.

- La formación educativa es una actividad emergente entre las personas mayores: el $86 \%$ de las personas mayores no han superado los estudios primarios, si bien se están incorporando a alguna actividad educativa de forma progresiva (4).

- La actividad física es una asignatura pendiente entre la población de edad. Si bien en forma de participación lúdica empieza a paliarse este déficit.

- El 45,5\% de las personas mayores destaca que goza de buena salud. Este porcentaje ha crecido 8 puntos y medio. Paralelamente, el porcentaje de las personas que afirman tener mala salud se ha reducido significativamente en el mismo periodo: del $24 \%$ al $14,5 \%$. Este es un indicador muy positivo aunque puesto en tela de juicio por los expertos (4).

\section{BIBLIOGRAFÍA}

1. INE/INEBASE. Proyecciones de población. "Censos de Población y Vivienda 2001". Disponible en: http://www.ine.es.

2. Tercer Informe sobre Demografía. Comisión Europea de Estadísticas (Eurostat). 2011. Disponible en http://ec.europa.eu/social/BlobServlet? docld $=6688$ \&langld $=$ en . 
3. Ministerio de Sanidad y Consumo. Instituto Nacional de Estadística. Encuesta Nacional de Salud (Período 2011-2012). Año 2013.

4. Libro Blanco del Envejecimiento Activo. Ministerio de Sanidad Política Social e Igualdad. Dirección General del IMSERSO. Madrid. 2011.

5. Libro Blanco de la Coordinación Sociosanitaria en España 2011. Ministerio de Sanidad Política Social e Igualdad. Dirección General del IMSERSO y Dirección General de la Agencia de Calidad del Sistema Nacional de Salud. Madrid. 2011.

6. Abellán García A, Vilches Fuentes J, Pujol Rodríguez R. "Un perfil de las personas mayores en España, 2014. Indicadores estadísticos básicos”. Madrid. Informes Envejecimiento en red n ${ }^{\circ} 6$ Disponible en: http://envejecimiento.csic.es/documentos/documentos/enred-indicadoresbasicos14. pdf. 2014.

7. Estrategia para el Abordaje de la Cronicidad en el Sistema Nacional de Salud. Estrategia aprobada por el Consejo Interterritorial del Sistema Nacional de Salud el 27 de junio de 2012. Ministerio de Sanidad, Servicios Sociales e Igualdad. Madrid, 2012.
8. Estrategia de Promoción de la Salud y Prevención en el Sistema Nacional de la Salud. En el marco del abordaje de la cronicidad en el SNS. Ministerio de Sanidad, Servicios Sociales e Igualdad. Madrid. 18 de diciembre de 2013.

9. Blasco Patiño F, Martínez López de Letona J, Villares P, Jiménez AI. El paciente anciano polimedicado: efectos sobre su salud y sobre el sistema sanitario. Información Terapéutica del Sistema Nacional de Salud 2005; 29:6.

10. Palop Larrea V, Martínez Mir I. Adherencia al tratamiento en el paciente anciano. Información Terapéutica del Sistema Nacional de Salud 2004; 28(5):113-20.

\section{CORRESPONDENCIA}

Primitivo Ramos Cordero

Agustín de Foxá, 31, $11^{a}$ planta dcha. 28036 Madrid

Email: primitivo.ramos@madrid.org 